\title{
Origin of Chaos in the Prometheus-Pandora System
}

\author{
Peter Goldreich \\ Institute for Advanced Study \\ Princeton NJ 08540 \\ E-mail: pmg@sns.ias.edu \\ \& \\ California Institute of Technology \\ Pasadena CA 91125 \\ E-mail:pmg@tapir.caltech.edu \\ and \\ Nicole Rappaport \\ Jet Propulsion Laboratory \\ California Institute of Technology \\ Pasadena CA 91109 \\ E-mail: Nicole.J.Rappaport@jpl.nasa.gov
}

Submitted to Icarus on June 18, 2003

Number of pages: 23

Number of tables: 3

Number of figures: 7 
Proposed Running Head: Origin of Chaos in the Prometheus / Pandora System

\section{Editorial correspondence to:}

Dr. Nicole J. Rappaport

Jet Propulsion Laboratory

MS 301-150

Pasadena CA 91109

Phone: (818) 354-8211

Fax: (818) 393-6388

E-mail: Nicole.J.Rappaport@jpl.nasa.gov 


\begin{abstract}
We demonstrate that the chaotic orbits of Prometheus and Pandora are due to interactions associated with the 121:118 mean motion resonance. Differential precession splits this resonance into a quartet of components equally spaced in frequency. Libration widths of the individual components exceed the splitting resulting in resonance overlap which causes the chaos. A single degree of freedom model captures the essential features of the chaotic dynamics. Mean motions of Prometheus and Pandora wander chaotically in zones of width $1.8 \mathrm{deg} \mathrm{yr}^{-1}$ and $3.1 \mathrm{deg} \mathrm{yr}^{-1}$, respectively.
\end{abstract}

Key Words: Satellites of Saturn, Orbits, Chaos 


\section{INTRODUCTION}

Goldreich and Rappaport (2003) (hereafter abbreviated as GR) showed that the motions of Prometheus and Pandora are chaotic. The chaos arises from their mutual gravitational interactions, which explains why their longitude discrepancies have comparable magnitudes and opposite signs (French et al. 2002). Numerical integrations that account for the full mutual interactions and Saturn's gravitational oblatenss yield a Lyapunov exponent of order $0.3 \mathrm{yr}^{-1}$. Although the results reported by GR assumed satellite masses based on a nominal density of $0.63 \mathrm{~g} \mathrm{~cm}^{-3}$, the Lyapunov exponent is insensitive to the assumed density above a critical value of approximately $0.3 \mathrm{~g} \mathrm{~cm}^{-3}$.

GR's integrations also reproduce qualitative features of the discrepancies between the longitudes of the satellites derived from analysis of recent HST data and predictions based on orbits fit to Voyager images (French et al. 2002). Sudden changes in the mean motions of Prometheus and Pandora are a striking feature of the numerical integrations. These occur at intervals of $6.2 \mathrm{yr}$ when the satellites' apses are anti-aligned. It is notable that the only clearly documented changes in the mean motions occurred around the time of the most recent apse anti-alignment $(c f . \mathrm{GR})$.

The plan of this paper is as follows. In $\S 2$ a quartet of 121:118 mean motion resonances is identified as the probable cause of the chaos. ${ }^{1}$ Then we describe two new programs in which interactions between the satellites are limited to those due to this quartet. The simpler of

\footnotetext{
${ }^{1}$ Differential apsidal precession splits each mean motion resonance into a multiplet of closely spaced components.
} 
these reduces the resonant dynamics to a system with one degree of freedom. Results from these programs are compared in $\S 3$ with those obtained from integrations that account for the full gravitational interactions. $\S 4$ is devoted to a discussion of the width of the chaotic zone.

\section{ORIGIN OF CHAOS}

\subsection{Resonant Quartet}

A systematic search for $j: j-k$ mean motion resonances with $k \leq 4$ turned up $j=121, \quad k=$ 3.

Following Murray and Dermott (2001), we write the disturbing function for the action of Pandora on Prometheus as

$$
\mathcal{R}=\frac{G m^{\prime}}{a^{\prime}} R_{D}
$$

and that for the action of Prometheus on Pandora as

$$
\mathcal{R}^{\prime}=\frac{G m}{a^{\prime}} R_{D}
$$

Here $m$ and $a$ denote mass and semi-major axis, and $G$ is the gravitational constant. ${ }^{2}$ To lowest order in the eccentricities, the terms in the literal expansion of the disturbing function associated with a $k=3$ resonance take the form

$$
R_{D}=e^{3} f_{82} \cos \left[121 \lambda^{\prime}-118 \lambda-3 \varpi\right]+
$$

\footnotetext{
${ }^{2}$ Primed and unprimed symbols refer to Prometheus and Pandora, respectively.
} 


$$
\begin{aligned}
& e^{2} e^{\prime} f_{83} \cos \left[121 \lambda^{\prime}-118 \lambda-2 \varpi-\varpi^{\prime}\right]+ \\
& e e^{\prime 2} f_{84} \cos \left[121 \lambda^{\prime}-118 \lambda-\varpi-2 \varpi^{\prime}\right]+ \\
& e e^{\prime 3} f_{85} \cos \left[121 \lambda^{\prime}-118 \lambda-3 \varpi^{\prime}\right],
\end{aligned}
$$

where $e, \lambda$, and $\varpi$ stand for eccentricity, mean longitude, and argument of periapse. The $f_{8 n}$ are expressed in terms of Laplace coefficients evaluated at $\alpha=a / a^{\prime} .^{3}$

$$
\begin{aligned}
f_{82}= & \frac{1}{48}\left\{\left(-26 j+30 j^{2}-8 j^{3}\right) b_{1 / 2}^{(j)}(\alpha)+\left(-9+27 j-12 j^{2}\right) \alpha \frac{d b_{1 / 2}^{(j)}(\alpha)}{d \alpha}+\right. \\
& \left.(6-6 j) \alpha^{2} \frac{d^{2} b_{1 / 2}^{(j)}(\alpha)}{d \alpha^{2}}-\alpha^{3} \frac{d^{3} b_{1 / 2}^{(j)}(\alpha)}{d \alpha^{3}}\right\}, \\
f_{83}=\quad & \frac{1}{16}\left\{\left(-9+31 j-30 j^{2}+8 j^{3}\right) b_{1 / 2}^{(j-1)}(\alpha)+\left(9-25 j+12 j^{2}\right) \alpha \frac{d b_{1 / 2}^{(j-1)}(\alpha)}{d \alpha}+\right. \\
& \left.(-5+6 j) \alpha^{2} \frac{d^{2} b_{1 / 2}^{(j-1)}(\alpha)}{d \alpha^{2}}+\alpha^{3} \frac{d^{3} b_{1 / 2}^{(j-1)}(\alpha)}{d \alpha^{3}}\right\}, \\
f_{84}= & \frac{1}{16}\left\{\left(8-32 j+30 j^{2}-8 j^{3}\right) b_{1 / 2}^{(j-2)}(\alpha)+\left(-8+23 j-12 j^{2}\right) \alpha \frac{d b_{1 / 2}^{(j-2)}(\alpha)}{d \alpha}+\right. \\
& \left.(4-6 j) \alpha^{2} \frac{d^{2} b_{1 / 2}^{(j-2)}(\alpha)}{d \alpha^{2}}-\alpha^{3} \frac{d^{3} b_{1 / 2}^{(j-2)}(\alpha)}{d \alpha^{3}}\right\}, \\
f_{85}=\quad & \frac{1}{48}\left\{\left(-6+29 j-30 j^{2}+8 j^{3}\right) b_{1 / 2}^{(j-3)}(\alpha)+\left(6-21 j+12 j^{2}\right) \alpha \frac{d b_{1 / 2}^{(j-2)}(\alpha)}{d \alpha}+\right. \\
& \left.(-3+6 j) \alpha^{2} \frac{d^{2} b_{1 / 2}^{(j-3)}(\alpha)}{d \alpha^{2}}+\alpha^{3} \frac{d^{3} b_{1 / 2}^{(j-3)}(\alpha)}{d \alpha^{3}}\right\} .
\end{aligned}
$$

Tables 1 and 2 list values for the parameters used in this paper. Satellite masses are given as fractions of Saturn's mass based on an assumed density of $0.63 \mathrm{~g} \mathrm{~cm}^{-3}$. Initial values for

\footnotetext{
${ }^{3}$ In the following, $j$ should be viewed as a shorthand for 121 .
} 
mean longitudes, apsidal angles, mean motions, and eccentricities are based on orbits fit to Voyager images by Jacobson (2001). Precession rates are calculated from the Saturnian gravitational field (Campbell and Anderson 1989).

Table 1. Masses, Initial Mean Longitudes, \& Mean Motions.

\begin{tabular}{|l|c|c|c|}
\hline Satellite & $m / M$ & Mean Longitude $\left(^{\circ}\right)$ & Mean Motion $\left(^{\circ} / s\right)$ \\
\hline Prometheus & $5.80 \times 10^{-10}$ & 188.53815 & $6.797331 \times 10^{-3}$ \\
Pandora & $3.43 \times 10^{-10}$ & 82.14727 & $6.629506 \times 10^{-3}$ \\
\hline
\end{tabular}

Table 2. Eccentricities, Initial Apsidal Angles, \& Precession Rates.

\begin{tabular}{|l|c|c|c|}
\hline Satellite & Eccentricity & Apsidal Angle $\left(^{\circ}\right)$ & Precession Rate $(\% / s)$ \\
\hline Prometheus & $2.29 \times 10^{-3}$ & 212.85385 & $3.1911 \times 10^{-5}$ \\
Pandora & $4.37 \times 10^{-3}$ & 68.22910 & $3.0082 \times 10^{-5}$ \\
\hline
\end{tabular}

Rates of change of the arguments, corresponding periods, and coefficients for the four terms in equation (3) are given in Table 3.

Table 3. Resonance Arguments, Rates of Change, Periods, and Coefficients. 


\begin{tabular}{|l|c|c|c|}
\hline Argument & Rate of Change $(\% / s)$ & Period (year) & Coefficient \\
\hline $121 \lambda^{\prime}-118 \lambda-3 \varpi$ & $-1.058 \times 10^{-5}$ & 1.078 & $e^{3} f_{82}=-0.001$ \\
$121 \lambda^{\prime}-118 \lambda-2 \varpi-\varpi^{\prime}$ & $-0.875 \times 10^{-5}$ & 1.303 & $e^{2} e^{\prime} f_{83}=0.006$ \\
$121 \lambda^{\prime}-118 \lambda-\varpi-2 \varpi^{\prime}$ & $-0.692 \times 10^{-5}$ & 1.648 & $e e^{\prime 2} f_{84}=-0.01$ \\
$121 \lambda^{\prime}-118 \lambda-3 \varpi^{\prime}$ & $-0.509 \times 10^{-5}$ & 2.239 & $e^{\prime 3} f_{85}=0.007$ \\
\hline
\end{tabular}

\section{$2.2 \quad$ Numerical Integrations}

To demonstrate that the quartet of $121: 118$ resonances is the cause of chaos in the Prometheus Pandora system, we develop two new programs to integrate the satellites' equations of motion. Interactions between the satellites are restricted to the four resonant interaction terms in the Fourier expansion of the disturbing function $R_{D}$. Each program evolves propagates the satellites' orbital elements rather than their cartesian coordinates and velocities as is done by the "old program" FSHEP used in GR.

We adopt epicyclic elliptic elements instead of the more standard osculating elliptic elements since, unlike the latter, they do not require short period terms to describe elliptic orbits around oblate planets (cf. Borderies-Rappaport and Longaretti 1994; henceforth, referred to as BRL.). BRL derive a modified version of Gauss' equations for the elements $a_{e}$, $e_{e}, \varpi_{e}=\omega_{e}+\Omega_{e}$, and $\lambda_{e}=\varpi_{e}+M_{e} \cdot{ }^{4}$ From these, it is a straightforward exercise to derive a restricted version of Lagrange's equations that is valid in the planar case. We work with a simplified set appropriate for low eccentricity orbits about a modestly oblate planet. The

\footnotetext{
${ }^{4}$ Hereafter we drop the subscript $e$.
} 
equations read:

$$
\begin{aligned}
& \frac{d \lambda}{d t}=\Omega, \\
& \frac{d a}{d t}=\frac{2}{\kappa a} \frac{\partial \mathcal{R}}{\partial \lambda}, \\
& \frac{d \varpi}{d t}=\Omega-\kappa, \\
& \frac{d e}{d t}=-\frac{1}{\kappa a^{2} e} \frac{\partial \mathcal{R}}{\partial \varpi},
\end{aligned}
$$

where

$$
\begin{aligned}
& \Omega^{2}=\frac{G M}{a^{3}}\left[1+\frac{3}{2}\left(\frac{R_{p}}{a}\right)^{2} J_{2}-\frac{15}{8}\left(\frac{R_{p}}{a}\right)^{4} J_{4}+\frac{35}{16}\left(\frac{R_{p}}{a}\right)^{6} J_{6} \quad \ldots\right] \\
& \kappa^{2}=\frac{G M}{a^{3}}\left[1-\frac{3}{2}\left(\frac{R_{p}}{a}\right)^{2} J_{2}+\frac{45}{8}\left(\frac{R_{p}}{a}\right)^{4} J_{4}-\frac{175}{16}\left(\frac{R_{p}}{a}\right)^{6} J_{6} \quad \ldots\right] .
\end{aligned}
$$

\subsection{New Programs}

In the planar approximation, the Prometheus-Pandora system has four degrees of freedom and preserves two integrals, total energy and total angular momentum. Thus each phase space trajectory lies on a six dimensional hypersurface embedded in the eight dimensional phase space.

FSHEPRES integrates the four, first-order equations (8)-(11) for each satellite. Thus it differs from FSHEP mainly because it limits the interactions between the satellites to 
resonant terms. ${ }^{5}$ Other minor differences arise because FSHEPRES integrates a simplified set of Lagrange's equations. In particular, the conservation laws are only approximately satisfied.

FSHEPSIM integrates only the first two equations (8) and (9) for each satellite. This drastic simplification is reasonable because, as a consequence of the rapid differential precession caused by Saturn's oblateness, interactions between the satellites produce negligible effects on their apsidal angles and orbital eccentricities (GR). A further simplification arises because conservation of energy implies ${ }^{6}$

$$
\frac{m}{a^{2}} \frac{d a}{d t}=\frac{m^{\prime}}{a^{\prime 2}} \frac{d a^{\prime}}{d t}
$$

Thus the resonant dynamics of the Prometheus-Pandora system reduces to a single degree of freedom system. It proves convenient to define the variable

$$
\psi=121 \lambda^{\prime}-118 \lambda,
$$

so that $R_{D}$ is expressed as

$$
R_{D}=\sum_{k=1}^{4} C_{k} \cos \left(\psi+\delta_{k}\right)
$$

with each $\dot{\delta}_{k}=$ constant. The evolution of $\psi$ is governed by

$$
\begin{aligned}
\frac{d^{2} \psi}{d t^{2}} & =3\left[\left(121 \Omega^{\prime}\right)^{2} \frac{m}{M}+\alpha(118 \Omega)^{2} \frac{m^{\prime}}{M}\right] \sum_{k=1}^{4} C_{k} \sin \left(\psi+\delta_{k}\right) \\
& =3 \times\left(121 \Omega^{\prime}\right)^{2} \frac{m}{M}\left[1+\alpha\left(m^{\prime} / m\right)\right] \sum_{k=1}^{4} C_{k} \sin \left(\psi+\delta_{k}\right)
\end{aligned}
$$

\footnotetext{
${ }^{5}$ We view as an unimportant difference the use of orbital elements by FSHEPRES and cartesian positions and velocities by FSHEP.

${ }^{6}$ In the following equation we ignore the interaction energy which is only significant near conjunctions.
} 
where in writing the second form of equation (18), we have applied the mean motion resonance relation $\Omega^{\prime} / \Omega \approx 118 / 121$ and emphasized the contribution from the lighter body, $m^{\prime}$. Individual mean longitudes follow from the relations

$$
\begin{aligned}
\lambda(t) & =\frac{-\alpha\left(m^{\prime} / m\right) \psi(t)+118[\lambda(0)+\dot{\lambda}(0) t]+121 \alpha\left(m^{\prime} / m\right)\left[\lambda^{\prime}(0)+\dot{\lambda}^{\prime}(0) t\right]}{121\left[1+\alpha\left(m^{\prime} / m\right)\right]}, \\
\lambda^{\prime}(t) & =\frac{\psi(t)+121 \alpha\left(m^{\prime} / m\right)\left[\lambda^{\prime}(0)+\dot{\lambda}^{\prime}(0) t\right]+118[\lambda(0)+\dot{\lambda}(0) t]}{121\left[1+\alpha\left(m^{\prime} / m\right)\right]} .
\end{aligned}
$$

Although we are left with a system described by a single degree of freedom, the absence of an energy integral still allows for chaos.

\section{COMPARISON OF RESULTS}

In this section we compare result obtained using FSHEPRES and FSHEPSIM with those obtained with FSHEP. As in GR, all our simulations are initialized with orbital elements for Prometheus and Pandora taken from Jacobson's ephemerides at epoch 1981 August 23 04:02:12 UTC. Comparisons among similar calculations done with each of the three programs are presented in Figures 1-6. As a consequence of chaos, qualitative similarities are the best that can be expected. These are apparent in each set of figures. However, there is a hint that FSHEPSIM yields a slightly smaller Lyapunov exponent than either FSHEP or FSHEPRES.

The similarity between the 20 year runs of longitude variations displayed Figures 1 and 2, while consistent with a Lyapunov exponent of approximately $0.3 \mathrm{yr}^{-1}$ as shown in Figure 3, probably also reflects the fact that at the Voyager epoch the mean motions of Prometheus 
and Pandora were close to their respective maximum and minimum. This accounts for the negative values of the rates of each resonant argument quoted in Table 3.

Figure 4 shows that over 3000 years the net variation of $121 \lambda^{\prime}-118 \lambda-2 \varpi^{\prime}-\varpi$ is much smaller than that of the other phases. Together with the constraint imposed by the conservation of energy on relative variations of $n$ and $n^{\prime}$, this implies that over this time interval the average mean value of $n$ was smaller than its initial value by about $0.67 \mathrm{deg}_{\mathrm{yr}}^{-1}$ and that of $n^{\prime}$ was larger by about $1.14 \mathrm{deg}_{\mathrm{yr}^{-1}}$. The relatively small value of $121 n^{\prime}-$ $118 n-2 \dot{\varpi}^{\prime}-\dot{\varpi}$ has a plausible dynamical explanation. The term with this phase rate is the one with the largest amplitude. Moreover, the amplitudes of terms with neighboring phase rates are about half as large and have opposite signs to that of the dominant term, whereas the amplitude of the term with the slowest phase rate is much smaller. Support for this explanation is provided by observing that interchanging the values of $e$ and $e^{\prime}$ results in the phase $121 \lambda^{\prime}-118 \lambda-\varpi^{\prime}-2 \varpi$ assuming the special status of being the one with the smallest net variation.

Figures 5 and 6 display longitude variations over 3000 years relative to the longitude based on the average mean motion over this interval. These are seen to be bounded by \pm 180 degrees.

\section{Discussion}

A closer examination of the one-degree of freedom model developed for FSHEP provides additional insight regarding chaos in the Prometheus-Pandora system. 
Overlapping resonances are known to produce chaos. Frequencies of individual members of the resonant quartet are spaced by $\dot{\varpi}-\dot{\varpi}^{\prime} \approx 1.0 \mathrm{rad} \mathrm{yr}^{-1}$. This is smaller than the half widths of the individual resonance components. ${ }^{7}$ Half widths computed from equation (18) and the data in Tables 1-3 are, in order of increasing resonance frequency, $1.5,3.7,5.1,4.1 \mathrm{rad} \mathrm{yr}^{-1}$.

Figure 7 shows surfaces of section based on data from 3000 year integrations using FSHEPSIM. A single point with coordinates $\psi-\varpi-2 \varpi^{\prime}, \dot{\psi}-\dot{\varpi}-2 \dot{\varpi}^{\prime}$ is plotted each time the apses align (every $6.2 \mathrm{yr}$ when $\varpi-\varpi^{\prime}=0$ modulo $2 \pi$ ). ${ }^{8}$ Nominal values for the satellites' masses were used for the upper panel. The scattering of points over an area in the phase plane is a signature of chaos. The balance in the number of points above and below the horizontal axis and the overall vertical width of their distribution are a consequence of the dominance of the resonance component with phase $121 \lambda^{\prime}-118 \lambda-\varpi-2 \varpi^{\prime}$. Satellite masses were reduced by a factor 10 below their nominal values to obtain the integrable example whose surface of section is shown in the lower panel.

Variations of $n$ and $n^{\prime}$ are related to those of $\dot{\psi}$ by

$$
\begin{aligned}
\frac{d n}{d t} & =\frac{1}{121\left[1+\alpha\left(m^{\prime} / m\right)\right]} \frac{d \psi}{d t} \\
\frac{d n^{\prime}}{d t} & =\frac{-\alpha\left(m^{\prime} / m\right)}{118\left[1+\alpha\left(m^{\prime} / m\right)\right]} \frac{d \psi}{d t}
\end{aligned}
$$

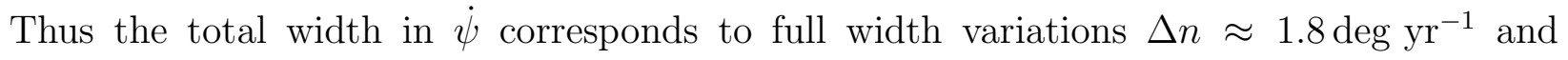

\footnotetext{
${ }^{7}$ The half width is the maximum angular velocity achieved during motion on the separatrix.

${ }^{8}$ We chose apse alignment to minimize the effects of the interaction energy.
} 
$\Delta n^{\prime} \approx 3.1 \operatorname{deg} \mathrm{yr}^{-1}$.

\section{Acknowledgments}

This research was supported by NASA Planetary Geology and Geophysics grant 344-30-53-02 and by NSF grant AST-0098301. 


\section{$6 \quad$ References}

Borderies-Rappaport N., and P.-Y. Longaretti 1994: Test Particle Motion around an Oblate Planet, Icarus 107, 129-141.

Campbell, J.K., and J.D. Anderson 1989: Gravity Field of the Saturnian System from Pioneer and Voyager Tracking Data, Astron. J. 97, 1485-1495.

French, R.G., C.A. McGhee, L. Dones, and J.J. Lissauer 2003: Saturn's wayward shepherds: the perigrinations of Prometheus and Pandora, Icarus 162, 144-171.

Goldreich, P. and N. Rappaport 2003: Chaotic Motions of Prometheus and Pandora, Icarus 162, 391-399.

Jacobson 2001: Tables of Prometheus and Pandora Planetocentric Mean Elements at

Julian Ephemeris Date 2444839.6682 Referred to the Earth Mean Equator and Equinox of J2000 System, Personal Communication.

Murray, C.D., and S.F. Dermott 2001: Solar System Dynamics, Cambridge University Press, 592 pp. 


\section{$7 \quad$ Figure Captions}

FIGURE 1: Prometheus longitude in degrees from numerical integration as a function of time over 20 years. A drift based on the initial mean motion has been subtracted from the longitudes. Dashed lines indicate the times of periapsis antialignment. Results shown in the top, middle, and bottom panels were obtained with the programs FSHEP, FSHEPRES, and FSHEPSIM.

FIGURE 2: Pandora longitude in degrees from numerical integration as a function of time over 20 years. A drift based on the initial mean motion has been subtracted from the longitudes. Dashed lines indicate the times of periapsis antialignment. Results shown in the top, middle, and bottom panels were obtained with the programs FSHEP, FSHEPRES, and FSHEPSIM.

FIGURE 3: Lyapunov exponent in $\mathrm{yr}^{-1}$ for the Prometheus-Pandora system over a period of $3 \times 10^{3}$ years. The results shown in the top, middle, and bottom panels were obtained with the programs FSHEP, FSHEPRES, and FSHEPSIM.

FIGURE 4: Phases $\psi+\delta_{k}, k=1, \ldots, 4$, in radians along the solution. Results shown in the top, middle, and bottom panels were obtained with the programs FSHEP, FSHEPRES, and FSHEPSIM.

FIGURE 5: Prometheus longitude in degrees from numerical integration as a function of time over 3000 years. A drift based on the mean motion averaged over 3000 years has been subtracted from the longitude. Results shown in the top, middle, and bottom panels were obtained with the programs FSHEP, FSHEPRES, and FSHEPSIM.

FIGURE 6: Pandora longitude in degrees from numerical integration as a function of time over a 3000 years. A drift based on the mean motion averaged over 3000 years has been subtracted from the longitude. Results shown in the top, middle, and bottom panels were obtained with the programs FSHEP, FSHEPRES, and FSHEPSIM.

FIGURE 7: Surfaces of section obtained by plotting $\left(\psi-\varpi-2 \varpi^{\prime}, \dot{\psi}-\dot{\varpi}-2 \dot{\varpi}^{\prime}\right)$ at each time of periapsis alignment over 3,000 years. Units are radians and radians per year. Computations were made with FSHEPSIM, for the top panel with the nominal value of $0.63 \mathrm{~g} \mathrm{~cm}^{-3}$ for the satellite density. For the bottom panel, the density was reduced by a factor of 10 in order to obtain an integrable example to contrast with the chaotic one shown above. 
This figure "icarusf1.gif" is available in "gif" format from: http://arxiv.org/ps/astro-ph/0307259 
This figure "icarusf2.gif" is available in "gif" format from: http://arxiv.org/ps/astro-ph/0307259 
This figure "icarusf3.gif" is available in "gif" format from: http://arxiv.org/ps/astro-ph/0307259 
This figure "icarusf4.gif" is available in "gif" format from: http://arxiv.org/ps/astro-ph/0307259 
This figure "icarusf5.gif" is available in "gif" format from: http://arxiv.org/ps/astro-ph/0307259 
This figure "icarusf6.gif" is available in "gif" format from: http://arxiv.org/ps/astro-ph/0307259 
This figure "icarusf7.gif" is available in "gif" format from: http://arxiv.org/ps/astro-ph/0307259 